\title{
Facile Method of Quinoxaline Synthesis Using Phenol as a New, Efficient and Cheap Catalyst at Room Temperature
}

\author{
Sami Sajjadifar ${ }^{1,3, *}$, Mohammad Ali Zolfigol², Seyed Ahmad Mirshokraie ${ }^{3}$, Sara Miri ${ }^{3}$, Omid Louie $^{3}$, \\ Eshagh Rezaee Nezhad ${ }^{3}$, Saaid Karimian ${ }^{3}$, Ghasem Darvishi $^{3}$, Elmira Donyadari ${ }^{2}$, Shohreh Farahmand $^{2}$ \\ ${ }^{1}$ Department of Chemistry, Faculty of Science, Ilam University, P.O. Box 69315516, Ilam, Iran \\ ${ }^{2}$ Faculty of Chemistry, Bu-Ali Sina University, Hamedan, P.O. Box 6517838683, Iran \\ ${ }^{3}$ Department of Chemistry, Payame Noor University, PO BOX 19395-4697 Tehran, Iran
}

\begin{abstract}
Abstarct A simple, highly efficient and green procedure for the condensation of aryl and alkyl 1,2-diamines with $\alpha$-diketones in the presence of catalytic amount of phenol $(20 \mathrm{~mol} \%)$ at room temperature is described. Using this method, quinoxaline derivatives as biologically interesting compounds are produced in high to excellent yields and short reaction times.
\end{abstract}

Keywords Quinoxaline, Phenol, 1,2-Diamine, A-Diketone, Green Chemistry

\section{Introduction}

Quinoxaline derivatives are a very important class of nitrogen-containing compounds and have been widely used in dyes[1] pharmaceuticals[2-3] and electrical/photochemical materials[4-9]. Quinoxaline ring moiety constitute part of the chemical structures of various antibiotics such as Echinomycin, Levomycin and Actinoleutin[10-11] that are known to inhibit growth of gram positive bacteria and are active against various transplantable tumors. A number of synthetic strategies have been developed for the preparation of substituted quinoxalines[12-14]. By far, the most common method relies on the condensation of an aryl 1,2-diamine with a 1,2-dicarbonyl compound in refluxing ethanol or acetic acid for $2-12 \mathrm{~h}$ giving $34-85 \%$ yields[15]. Recently, Heravi et al.[16] and More et al.[17] reported greener methods for the synthesis of quinoxaline derivatives in green solvents $\left(\mathrm{EtOH} / \mathrm{H}_{2} \mathrm{O}\right)$, using copper sulphate pentahydrate and cerium (IV) ammonium nitrate as catalysts, respectively. 2,3-Disubstituted quinoxalines have also been prepared by Suzuki-Miyaura coupling reaction[18], condensation of o-phenylenediamines and 1,2-dicarbonyl compounds in $\mathrm{MeOH} / \mathrm{AcOH}$ under microwave irradiation[19], iodine catalyzed cyclocondensation of 1,2- dicarbonyl compounds and substituted o-phenylene diamines in DMSO[20], $\mathrm{CH}_{3} \mathrm{CN}$ [21]. Different catalysts used for quinoxaline synthesis such as IBX[22], Oxalic Acid[23], SBSSA[24], Microwave $/ \mathrm{I}_{2}[25], \mathrm{SnCl}_{2} / \mathrm{SiO}_{2}$ [26], $\mathrm{I}_{2}$ [27], Ultrasound Irradiaton[28], $\mathrm{NH}_{4} \mathrm{Cl}[29],\left(\mathrm{NH}_{4}\right)_{6} \mathrm{Mo}_{7} \mathrm{O}_{24} \cdot 4 \mathrm{H}_{2} \mathrm{O}$ [30], Citric

* Corresponding author:

ss_sajjadifar@yahoo.com (Sami Sajjadifar)

Published online at http://journal.sapub.org/ajoc

Copyright (C) 2012 Scientific \& Academic Publishing. All Rights Reserved acid[31], ionic liquid[32], Bentonit Clay K-10[33], AcOH[3 4] and BSA[35]. We disclose herein our results for the synthesis of quinoxalines using catalytic amounts of phenol in water as an acidic solution at room temperature.

\section{Methods}

\subsection{General}

IR spectra of the compounds were obtained on a Shimadzu IR-435 spectrometer using a $\mathrm{KBr}$ disk. The ${ }^{1} \mathrm{H}$ nuclear magnetic resonance $\left({ }^{1} \mathrm{H}\right.$ NMR) spectra were recorded on a Bruker AQS 300 Avance instrument at $300 \mathrm{MHz}$ in dimethyl sulfoxide $\left(\mathrm{DMSO}_{\mathrm{C}} \mathrm{d}_{6}\right.$ ) using tetramethylsilane as an internal standard. The progress of reaction was followed with thin-layer chromatography (TLC) using silica gel SILG/UV 254 and 365 plates. All the products are known compounds and were characterized by comparing the IR, ${ }^{1} \mathrm{H}$ NMR, and ${ }^{13} \mathrm{C}$ NMR spectroscopic data and their melting points with the literature values.

\subsection{General Procedure of Preparation of Quinox-Alines}

A solution of aromatic o-diamine $(1 \mathrm{mmol})$ and a 1,2-dicarbonyl compound (1 $\mathrm{mmol})$ in ethanol:water $(7: 3,10$ $\mathrm{mL}$ ) was stirred at room temperature in the presence of catalytic amount of phenol $(20 \mathrm{~mol} \%, 0.01 \mathrm{~g})$. The progress of the reaction was monitored by TLC (n-hexan-ethylacetate 20:1). After completion of the reaction, water $(20 \mathrm{~mL})$ added to the mixture and was allowed to stand at room temperature for $30 \mathrm{~min}$. During this time, crystals of the pure product were formed which were collected by filtration and dried. For further purification, the products recrystallized from hot ethanol. 


\subsection{Selected Specteral Data}

\subsubsection{2,3-Diphenylquinoxaline(1Q)}

white solid m.p 125-127[lit. 128-129] $]^{22}$, FT-IR (KBr): $1556 \mathrm{~cm}^{-1} ;{ }^{1} \mathrm{H}-\mathrm{NMR} \quad\left(\mathrm{FT}-300 \mathrm{MHz}, \mathrm{CDCl}_{3} / \mathrm{TMS}\right)$ : dppm7.33977(bs, 6H, Ar-H) 7.54183(bs, 4H, Ar-H) 7.74584( bs, 2H, Ar-H) 8.20007( bs, $2 \mathrm{H}, \mathrm{Ar}-\mathrm{H}) ;{ }^{13} \mathrm{C} \mathrm{NMR}$ (300 MHz, $\mathrm{CDCl}_{3}$ ): 128.290, 128.896, 129.121, 129.913, 130.066, 138.921, 141.115, 153.384; MS: $m / z=282(\mathrm{M}+)$.

\subsubsection{6-Methyl-2,3-diphenylquinoxaline(2Q)}

brown solid m.p 113-115[lit. 116-117] $]^{24}$, FT-IR (KBr): $1619 \mathrm{~cm}^{-1}$ (stretching $\left.\mathrm{C}=\mathrm{N}\right)$; ${ }^{1} \mathrm{H}-\mathrm{NMR} \quad(\mathrm{FT}-300 \mathrm{MHz}$, $\mathrm{CDCl}_{3} / \mathrm{TMS}$ ): dppm 2.61(s, 3H, Ar- $\left.\mathrm{CH}_{3}\right)$ 7.35( s, 6H, Ar-H) 7.55(d, J=6.48, 4H, Ar-H)7.60(s, 1H, Ar-H) 7.98(s, 1H, Ar-H) $8.09(\mathrm{~d}, \mathrm{~J}=8.4,1 \mathrm{H}, \mathrm{Ar}-\mathrm{H}) ;{ }^{13} \mathrm{C}$ NMR $(300 \mathrm{MHz}$, $\left.\mathrm{CDCl}_{3}\right)$ : 21.948, 128.040, 128.244, 128.663, 128.731, $129.903,129.915,132.321,139.246,139.728,140.486$, 141.289, 152.552, 153.289; MS: $m / z=296(\mathrm{M}+)$.

\subsubsection{6-Nitro-2,3-diphenylquinoxaline(5Q)}

red solid m.p 185-187[lit. 185-187] ${ }^{24}$, FT-IR (KBr): 1656 $\mathrm{cm}^{-1}$ (stretching $\left.\quad \mathrm{C}=\mathrm{N}\right) ; \quad{ }^{1} \mathrm{H}-\mathrm{NMR} \quad(\mathrm{FT}-300 \quad \mathrm{MHz}$, $\mathrm{CDCl}_{3} / \mathrm{TMS}$ ): dppm 7.38(bs, 6H, Ar-H) 7.56(bs, 4H, Ar-H) 8.28(bs, 1H, Ar-H) 8.45(bs, 1H, Ar-H) 9.02(bs, 1H, Ar-H); ${ }^{13} \mathrm{C}$ NMR (300 MHz, $\left.\mathrm{CDCl}_{3}\right):$; MS: 123.269, 125.512, $128.450,129.667,129.854,129.953,130.666,137.9950$, $139.870,143.390,147.801,155.621,156.176$; MS: $m / z=$ $327(\mathrm{M}+)$.

\subsubsection{2,3-bis(4-Methoxyphenyl)quinoxaline(8Q)}

yellow solid m.p 134-136[lit. 148-150] $]^{36}$, FT-IR (KBr): $1615 \mathrm{~cm}^{-1}$ (stretching $\left.\mathrm{C}=\mathrm{N}\right)$; ${ }^{1} \mathrm{H}-\mathrm{NMR} \quad(\mathrm{FT}-300 \mathrm{MHz}$, $\mathrm{CDCl}_{3} / \mathrm{TMS}$ ): dppm 3.85(s, 6H, $\left.2 \times \mathrm{CH}_{3}\right) 6.87(\mathrm{~d}, \mathrm{~J}=7.77,1 \mathrm{H}$, Ar-H) 6.94 (d, J=7.77, 4H, Ar-H) 7.50 (d, J=7.14, 1H, Ar-H) 7.71( s, 1H, Ar-H) 7.93(d, J=7.62, 4H, Ar-H) 8.13( s, 1H, $\mathrm{Ar}-\mathrm{H}) ;{ }^{13} \mathrm{C} \mathrm{NMR}\left(300 \mathrm{MHz}, \mathrm{CDCl}_{3}\right)$ : 55.291, 55.615, $113.786,114.300,126.271,128.875,129.645,131.307$, $131.449,132.315,140.867,152.926,160.267,164.867$, 193.506; MS: $m / z=342(\mathrm{M}+)$.

\subsubsection{Dibenzo[a,c]phenazine(15Q)}

yellow solid m.p 224-226[lit. 223-225] $]^{21}$, FT-IR (KBr): $1604 \mathrm{~cm}^{-1}$ (stretching $\left.\mathrm{C}=\mathrm{N}\right) ;{ }^{1} \mathrm{H}-\mathrm{NMR} \quad(\mathrm{FT}-300 \mathrm{MHz}$, $\left.\mathrm{CDCl}_{3} / \mathrm{TMS}\right)$ : dppm 7.71(s, 4H, Ar-H) 7.85(s, 2H, Ar-H) 8.35(s, 2H, Ar-H) 8.43(s, 2H, Ar-H) 9.33(s, 2H, Ar-H); ${ }^{13} \mathrm{C}$ NMR (300 MHz, $\left.\mathrm{CDCl}_{3}\right): 122.875,126.448,128.023$, $128.925,129.356,130.205,130.661,132.019,141.323$, 141.876; MS: $m / z=280(\mathrm{M}+)$.

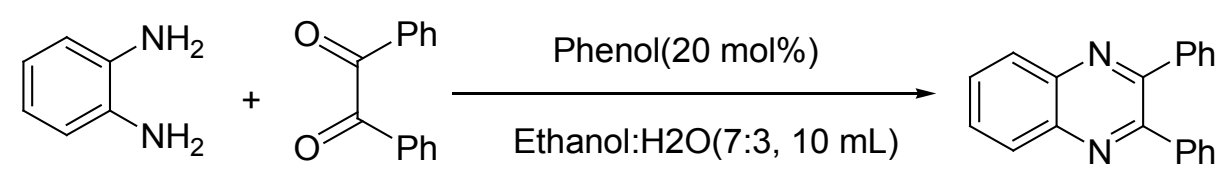

Scheme 1. 2,3-diphenylquinoxaline synthesis as a model by using phenol

\subsubsection{1-Methyl-dibenzo[a,c]phenazine(16Q)}

brown solid m.p 219-221[lit. 208-210] $]^{24}$, FT-IR (KBr): $1624 \mathrm{~cm}^{-1}$ (stretching $\mathrm{C}=\mathrm{N}$ ); ${ }^{1} \mathrm{H}-\mathrm{NMR}$ (FT-300 $\mathrm{MHz}$, $\left.\mathrm{CDCl}_{3} / \mathrm{TMS}\right): \operatorname{dppm} 2.65\left(\mathrm{~s}, 3 \mathrm{H}, \mathrm{CH}_{3}\right)$ 7.72(bs, 5H, Ar-H) 8.04(s, 1H, Ar-H) 8.15(s, 1H, Ar-H) 8.47(s, 2H, Ar-H) 9.32(bs, $2 \mathrm{H}, \mathrm{Ar}-\mathrm{H}) ;{ }^{13} \mathrm{C}$ NMR (300 MHz, $\left.\mathrm{CDCl}_{3}\right): 20.058$, $122.795,126.032,126.178,127.793,128.797,129.978$, $130.127,131.727,131.922,132.368,140.393,140.573$, 141.508, 141.971; MS: $m / z=294(\mathrm{M}+)$.

\subsubsection{1-Benzoil-dibenzo[a,c]phenazine(17Q)}

yellow solid m.p 245-247, FT-IR (KBr): 1653, 1606 $\mathrm{cm}^{-1}$ (stretching $\left.\mathrm{C}=\mathrm{N}\right)$; ${ }^{1} \mathrm{H}-\mathrm{NMR} \quad(\mathrm{FT}-300 \quad \mathrm{MHz}$, $\left.\mathrm{CDCl}_{3} / \mathrm{TMS}\right)$ : dppm 7.27(s, 1H, Ar-H) 7.60(s, 2H, Ar-H) 7.71(s, 2H, Ar-H) 7.79(s, 2H, Ar-H) 7.98(s, 2H, Ar-H) 8.35(s, 1H, Ar-H) 8.52(s, 3H, Ar-H) 8.69(s, 1H, Ar-H) 9.31(s, 1H, Ar-H) 9.44(s, 1H, Ar-H); ${ }^{13} \mathrm{C}$ NMR (300 MHz, $\left.\mathrm{CDCl}_{3}\right)$ : 123.048, 128.254, 128.601, 129.450, 130.220, $130.754,131.056,132,916,140.540,140.980,141.323$, 141.876, 154.046; MS: $m / z=384(\mathrm{M}+)$.

\subsubsection{2-Bromopyrido-[2,3-b]dibenzo[5,6-7,8]quinoxaline(1 8Q)}

yellow solid m.p 216-218 ${ }^{\circ} \mathrm{C}$, FT-IR (KBr): 1603 $\mathrm{cm}^{-1}$ (stretching $\left.\mathrm{C}=\mathrm{N}\right) ; \quad{ }^{1} \mathrm{H}-\mathrm{NMR} \quad(\mathrm{FT}-300 \quad \mathrm{MHz}$, $\mathrm{CDCl}_{3} / \mathrm{TMS}$ ): dppm 7.72(bs, 4H, Ar-H) 8.41(bs, 2H, Ar-H) 8.66(s, 1H, Ar-H) 9.06(s, 1H, Ar-H) 9.12(s, 1H, Ar-H) 9.30(s, 1H, Ar-H); ${ }^{13} \mathrm{C}$ NMR (300 MHz, $\left.\mathrm{CDCl}_{3}\right): 122.858$, 126.519 , 127.292, 128.048, 129.026, 131.393, 139.507, 143.815, 155.170; MS: $m / z=360(\mathrm{MH}+)$.

\subsubsection{9-Methylacenaphto[1,2-b]quinoxaline(23Q)}

brown solid m.p 231-242[lit. $\left.>300{ }^{\circ} \mathrm{C}\right]^{24}$, FT-IR $(\mathrm{KBr})$ : $1626 \mathrm{~cm}^{-1}$ (stretching $\left.\mathrm{C}=\mathrm{N}\right) ;{ }^{1} \mathrm{H}-\mathrm{NMR} \quad(\mathrm{FT}-300 \mathrm{MHz}$, $\left.\mathrm{CDCl}_{3} / \mathrm{TMS}\right)$ : dppm 2.56(s, 3H, $\left.\mathrm{CH}_{3}\right) 7.49(\mathrm{~d}, \mathrm{~J}=8.31,1 \mathrm{H}$, Ar-H) 7.70(t, J=7.5, 2H, Ar-H) 7.88(s, 1H, Ar-H) 7.94(d, $\mathrm{J}=8.07,2 \mathrm{H}, \mathrm{Ar}-\mathrm{H}) \quad 7.99(\mathrm{~d}, \mathrm{~J}=8.61,1 \mathrm{H}, \mathrm{Ar}-\mathrm{H})$ 8.27(t, $\mathrm{J}=6.40$, $2 \mathrm{H}, \mathrm{Ar}-\mathrm{H}) ;{ }^{13} \mathrm{C} \mathrm{NMR}\left(300 \mathrm{MHz}, \mathrm{CDCl}_{3}\right): 21.735,121.583$, $121.785,128.484,128.554,128.938,129.113,129.284$, $129.799,131.250,131.645,136.097,139.359$, 139.666, 140.915, 153.035, 153.661; MS: $m / z=268(\mathrm{M}+)$.

\subsubsection{Acenaphto[1,2-b]quinoxaline(25Q)}

yellow solid m.p 241-242[lit. 238-240] ${ }^{24}$, FT-IR (KBr): $1614 \mathrm{~cm}^{-1}$ (stretching $\left.\mathrm{C}=\mathrm{N}\right) ;{ }^{1} \mathrm{H}-\mathrm{NMR} \quad(\mathrm{FT}-300 \mathrm{MHz}$, $\left.\mathrm{CDCl}_{3} / \mathrm{TMS}\right)$ : dppm 7.74-7.81(m, 4H, Ar-H) 8.05(d, J=8.16, $2 \mathrm{H}, \mathrm{Ar}-\mathrm{H})$ 8.20-8.23(m, 2H, Ar-H) 8.4(d, J=6.87, 2H, Ar-H); ${ }^{13} \mathrm{C}$ NMR (300 MHz, $\mathrm{CDCl}_{3}$ ): 122.328, 128.679, 129.285, 129.442, 129.694, 129.900, 131.248, 136.479, 140.707, 153.595; MS: $m / z=254(\mathrm{M}+)$. 


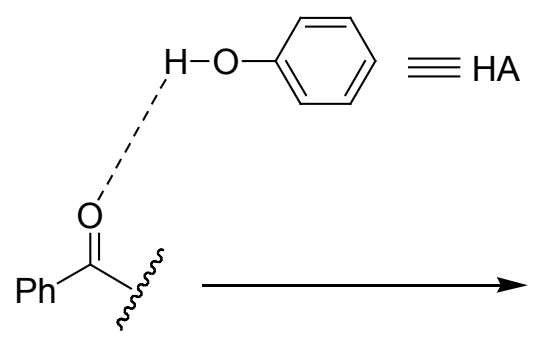<smiles>CCCCC(C)C(=O)Oc1ccccc1</smiles>

$\mathrm{H}_{2} \ddot{\mathrm{N}}$<smiles>C/C=C\N</smiles>

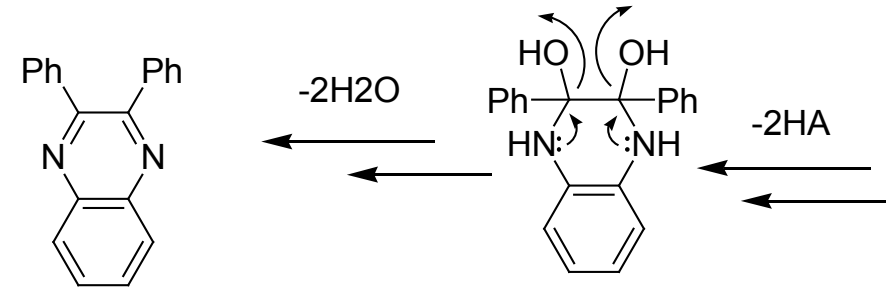

Scheme 2. Proposed mechanism of catalyst effect<smiles>CC(Nc1ccccc1N)C(C)(O)c1ccccc1</smiles><smiles>[Y]C=CC</smiles>

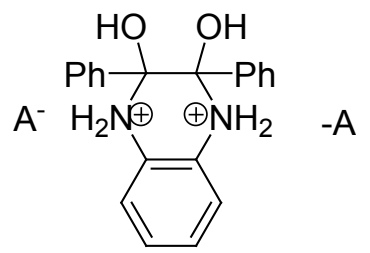

Table 1. Quinoxalines synthesis from 1,2-diamines and $\alpha$-diketones by using phenol (20 mol\%)

Entry


5<smiles>Nc1ccc([N+](=O)[O-])cc1N</smiles><smiles>O=C(C(=O)c1ccccc1)c1ccccc1</smiles><smiles>O=C(C(=O)c1ccccc1)c1ccccc1</smiles><smiles>Nc1ccc(Br)nc1N</smiles><smiles>Nc1cccnc1N</smiles><smiles>Nc1ccccc1N</smiles>

8<smiles>O=C(C(=O)c1ccccc1)c1ccccc1</smiles><smiles>Nc1ccc([N+](=O)[O-])cc1N</smiles><smiles>COc1ccc(C(=O)C(=O)c2ccc(OC)cc2)cc1</smiles>

10<smiles>Nc1ccc(Br)nc1N</smiles><smiles>COc1ccc(C(=O)C(=O)c2ccc(OC)cc2)cc1</smiles><smiles>COc1ccc(C(=O)C(=O)c2ccc(OC)cc2)cc1</smiles><smiles>Nc1ccncc1N</smiles>

11

12<smiles>Nc1cccnc1N</smiles><smiles>COc1ccc(C(=O)C(=O)c2ccc(OC)cc2)cc1</smiles><smiles>COc1ccc(C(=O)C(=O)c2ccc(OC)cc2)cc1</smiles>

13<smiles>Cc1ccc(N)c(N)c1</smiles><smiles>O=[N+]([O-])c1ccc2nc(-c3ccccc3)c(-c3ccccc3)nc2c1</smiles><smiles>Brc1ccc2nc(-c3ccccc3)c(-c3ccccc3)nc2n1</smiles><smiles>c1ccc(-c2nc3cccnc3nc2-c2ccccc2)cc1</smiles>

$03: 45$

$134-137$

$141-142[37]$<smiles>COc1ccc(-c2nc3ccccc3nc2-c2ccc(OC)cc2)cc1</smiles>

03:20

91

$134-136$

$148-150[22]$

03:00

90

$188-189$

----[26]

05:45

88

$124-126$

07:15

90

$120-122$

02:30

00:02

$131-134$

00:05

90

$123-125$

$129-131[21]$ 
14<smiles>Nc1ccc(C(=O)c2ccccc2)cc1N</smiles><smiles>Nc1ccccc1N</smiles>

15

16<smiles>Cc1ccc(N)c(N)c1</smiles>

17<smiles>Nc1ccc(C(=O)c2ccccc2)cc1N</smiles>

18<smiles>Nc1ccc(Br)nc1N</smiles><smiles>Nc1ccc([N+](=O)[O-])cc1N</smiles>

20<smiles>Nc1cccnc1N</smiles>

21<smiles>COc1ccc(C(=O)C(=O)c2ccc(OC)cc2)cc1</smiles><smiles>COc1ccc(-c2nc3ccc(C(=O)c4ccccc4)cc3nc2-c2ccc(OC)cc2)cc1</smiles><smiles>[124Os]</smiles>

$----[27]$<smiles>O=C1C(=O)c2ccccc2-c2ccccc21</smiles><smiles></smiles>
00:02

$225-226$

$223-225[21]$<smiles>O=C1C(=O)c2ccccc2-c2ccccc21</smiles><smiles>Cc1ccc2nc3c4ccccc4c4ccccc4c3nc2c1</smiles>

00:10 95

$218-220$ $208-210[21]$<smiles>O=C1C(=O)c2ccccc2-c2ccccc21</smiles><smiles>O=C(c1ccccc1)c1ccc2nc3c4ccccc4c4ccccc4c3nc2c1</smiles><smiles>Brc1ccc2nc3c4ccccc4c4ccccc4c3nc2n1</smiles>

00:45

86

$215-216$

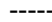

00:20

93

$259-260$

------

02:00

91

$221-223$

03:00

94

$159-162$ 
<smiles>Nc1ccc([N+](=O)[O-])cc1N</smiles><smiles>Cc1ccc(N)c(N)c1</smiles>

24<smiles>Nc1cccnc1N</smiles>

25<smiles>Nc1ccccc1N</smiles>

26<smiles>Nc1ccc(Br)nc1N</smiles>

27<smiles>Nc1ccc(C(=O)c2ccccc2)cc1N</smiles><smiles>O=C1C(=O)c2cccc3cccc1c23</smiles><smiles>O=C1C(=O)c2cccc3cccc1c23</smiles><smiles>O=C1C(=O)c2cccc3cccc1c23</smiles><smiles>O=C1C(=O)c2cccc3cccc1c23</smiles><smiles>O=C1C(=O)c2cccc3cccc1c23</smiles><smiles>CC1CCC(=O)C1=O</smiles><smiles>O=[N+]([O-])c1ccc2nc3c(nc2c1)-c1cccc2cccc-3c12</smiles>

04:30

93

321-323<smiles>Cc1ccc2nc3c(nc2c1)-c1cccc2cccc-3c12</smiles>

00:02

97

$228-229$

$>300[21]$

03:00

93

$229-231$

$00: 25$

91

235-236

238-240[21]

04:00<smiles>Brc1ccc2nc3c(nc2n1)-c1cccc2cccc-3c12</smiles>

94

224-226<smiles>CC1CCc2nc3ccc(C(=O)c4ccccc4)cc3nc2C1CCc1ccccc1</smiles>

\section{Result and Discussion}

Table 2. The condensation of benzene-1,2-diamine ( $1 \mathrm{mmol})$ with benzil $(1 \mathrm{mmol})$ in the presence of different catalysts $(0.2 \mathrm{mmol}, 20 \mathrm{~mol} \%)$ in EtOH:H2O (7:3) at room tempera

\begin{tabular}{|c|c|c|c|}
\hline Number & Catalyst name(cat\%) & $\begin{array}{c}\text { Time } \\
(\text { mine })\end{array}$ & $\begin{array}{c}\text { Yield\% } \\
{[\text { lit] }}\end{array}$ \\
\hline 1 & ---- & 600 & ---- \\
\hline $\mathbf{2}$ & Phenol(20 mol\%) & $\mathbf{1 0}$ & $\mathbf{9 8}$ \\
\hline 3 & Oxalic acid(20 mol\%) & 10 & $93[23]$ \\
\hline 4 & $\mathrm{ZnCl}_{2}(20 \mathrm{~mol} \%)$ & 240 & 70 \\
\hline 5 & $\mathrm{Mn}(\mathrm{OAc})_{2}(20 \mathrm{~mol} \%)$ & 240 & 78 \\
\hline 6 & $\mathrm{CoCl}_{2}(20 \mathrm{~mol} \%)$ & 240 & 81 \\
\hline 7 & $\mathrm{CuCl}_{2}((20 \mathrm{~mol} \%))$ & 240 & 69 \\
\hline 8 & $\mathrm{Ni}(\mathrm{OAc})_{2}(20 \mathrm{~mol} \%)$ & 240 & 68 \\
\hline 9 & $\mathrm{BSA}(3 \mathrm{~mol} \%)$ & 5 & $98[35]$ \\
\hline
\end{tabular}

In order to find a suitable catalyst for the synthesis of quinoxalines from 1,2-diamines and $\alpha$-diketones, the conden- sation of benzene-1,2-diamine with benzil was chosen as a model to provide compound 1Q (Scheme 1), and its behavior was studied in the presence of various catalysts in $\mathrm{EtOH} / \mathrm{H}_{2} \mathrm{O}$ at room temperature. The results are displayed in Table 1. As it can be seen from Table 2, phenol as an organic catalyst afforded the good results with respect to the inorganic catalysts. Total reactions are summarized in Table 1. Water is a desirable solvent for chemical reactions for reasons of cost, safety and environmental concerns, use of water in this reaction gave only moderate yields of products (67\% after $3 \mathrm{~h})$. For chosen better solvent, different solvents were examined (Table 3). $\mathrm{H}_{2} \mathrm{O}$ :Ethanol $(3: 7,10 \mathrm{~mL}$ ) was better solvent. The optimum yields of the products are obtained when $20 \mathrm{~mol} \%$ of phenol is used.o-Phenylenediam ines and 1,2-dicarbonyl compounds with electron-donating or electron-withdrawing groups were used. As indicated in the Table 1 both electron rich and electron deficient 1,2-dicarbonyl compounds worked pretty well, mostly leading to high yields of products but withdrawing groups had lower yield. 
Table 3. Solvent and catalyst optimize in synthesis 2,3-diphenylquinoxali ne as a model

\begin{tabular}{|c|c|c|c|c|}
\hline Number & Solvent & $\mathrm{Cat} \%$ & $\begin{array}{l}\text { Time } \\
(\mathrm{min})\end{array}$ & Yield \% \\
\hline 1 & $\mathrm{H} 2 \mathrm{O}(10 \mathrm{~mL})$ & 3 & 240 & 67 \\
\hline 2 & $\mathrm{H} 2 \mathrm{O}(10 \mathrm{~mL})$ & 5 & 180 & 76 \\
\hline 3 & $\mathrm{H} 2 \mathrm{O}(10 \mathrm{~mL})$ & 10 & 80 & 76 \\
\hline 4 & EtOH:H2O (1:1, $10 \mathrm{~mL})$ & 1 & 200 & 70 \\
\hline 5 & EtOH:H2O $(1: 1,10 \mathrm{~mL})$ & 5 & 150 & 75 \\
\hline 6 & EtOH:H2O (1:1, $10 \mathrm{~mL})$ & 10 & 55 & 78 \\
\hline 7 & EtOH:H2O $(4: 1,10 \mathrm{~mL})$ & 10 & 40 & 80 \\
\hline 8 & EtOH:H2O (4:1, $10 \mathrm{~mL})$ & 20 & 10 & 90 \\
\hline 9 & EtOH:H2O(7:3, 10mL) & 20 & 5 & 98 \\
\hline 10 & EtOH:H2O(7:3, 10mL) & 30 & 25 & 92 \\
\hline 11 & EtOH:H2O (9:1, $10 \mathrm{~mL})$ & 5 & 100 & 79 \\
\hline 12 & $\mathrm{EtOH}(10 \mathrm{~mL})$ & 10 & 60 & 81 \\
\hline 13 & Ethanol $(10 \mathrm{~mL})$ & 15 & 35 & 78 \\
\hline 14 & Ethanol $(10 \mathrm{~mL})$ & 20 & 20 & 85 \\
\hline
\end{tabular}

\section{Conclusions}

In summary, we have developed an efficient method for the synthesis of quinoxaline derivatives via the condensation of 1,2-diamines with $\alpha$-diketones. This new strategy has several advantages, such as excellent yield, mild reaction conditions, short duration of reaction time, low cost, simple experimental as well as isolation procedures, and finally, it is in agreement with the green chemistry protocols. These advantages will make this method become an attractive greener technique for the construction of quinoxalines and notably similar molecules, compared to the existing methods.

\section{ACKNOWLEDGEMENTS}

The authors gratefully acknowledge partial support of this work by Payame Noor University (PNU) of Ilam.

\section{REFERENCES}

[1] Brock, E. D.; Lewis, D. M.; Yousaf, T. I.; Harper, H. H. WO 9951688, 1999.

[2] Gazit, A.; App, H.; McMahon, G.; Chen, J.; Levitzki, A.; Bohmer, F. D. J. Med. Chem. 1996, 39, 2170.

[3] Sehlstedt, U.; Aich, P.; Bergman, J.; Vallberg, H.; Norden, B.; Graslund, A. J. Mol. Biol. 1998, 278, 31.

[4] Dailey, S.; Feast, J. W.; Peace, R. J.; Sage, I. C.; Till, S.; Wood, E. L. J. Mater. Chem. 2001, 11, 2238.
[5] O’Brien, D.; Weaver, M. S.; Lidzey, D. G.; Bradley, D. D. C. Appl. Phys. Lett. 1996, 69, 881.

[6] Yamamoto, T.; Sugiyama, K.; Kushida, T.; Inoue, T.; Kanbara, T. J. Am. Chem. Soc. 1996, 118, 3930.

[7] Yamamoto, T.; Zhou, Z.-H.; Kanbara, T.; Shimura, M.; Kizu, K.; Maruyama, T.; Nakamura, Y.; Fukuda, T.; Lee, B.-L.; Ooba, N.; Tomaru, S.; Kurihara, T.; Kanno, T.; Kubota, K.; Sasaki, S. J. Am. Chem. Soc. 1996, 118, 10389.

[8] Nurulla, I.; Yamaguchi, I.; Yamamoto, T. Polym. Bull. 2000, 44, 231.

[9] Yamamoto, T.; Lee, B.-L.; Kokubo, H.; Kishida, H.; Hirota, K.; Wakabayashi, T.; Okamoto, H. Macromol. Rapid Commun. 2003, 24, 440.

[10] Dell, A.; William, D. H.; Morris, H. R.; Smith, G. A.; Feeney, J.; Roberts, G. C. K. J. Am. Chem. Soc. 1975, 97, 2497.

[11] Bailly, C.; Echepare, S.; Gago, F.; Waring, M. J. Anti-Cancer Drug Des. 1999, 15, 291.

[12] Eicher, T.; Hauptmann, S. The Chemistry of Heterocycles; Thieme: New York, 1995; 417, 434.

[13] Barluenga, J.; Aznar, F.; Liz, R. Cabal, M.-P. Synthesis 1985, 313.

[14] Petukhov, P. A.; Tkachev, A. V. Tetrahedron 1997, 53, 9761.

[15] Brown, D. J. Quinoxalines: Supplement II. In The Chemistry of Heterocyclic Compounds; Taylor, E. C., Wipf, P., Eds.; John Wiley\& Sons: New Jersey, 2004.

[16] M.M. Heravi, S. Taheri, K. Bakhtiari, et al. Catal. Commun. 8 (2007) 211.

[17] S.V. More, M.N.V. Sastry, C.F. Yao, Green Chem. 8 (2006) 91.

[18] Mao, L. Sakurai, H. Hirao, T. Synthesis 2004, 2535.

[19] Zhao, Z.; Wisnoski, D. D.; Wolkenberg, S. E.; Leister, W. H.; Wang, Y.; Lindsley, C. W. Tetrahedron Lett. 2004, 45, 4873.

[20] Bhosale, R. S.; Sarda, S. R.; Ardhapure, S. S.; Jadhav, W. N.; Bhusare, S. R.; Pawar, R. P. Tetrahedron Lett. 2005, 46, 7183.

[21] More, S. V.; Sastry, M. N. V.; Wang, C. -C.; Yao C.-F. Tetrahedron Lett. 2005, 46, 6345.

[22] Heravi, M. M.; Bakhtiari, K.; Tehrani, M. H.; Javadi, N. M.; Oskooie, H. A.; Arkivoc 2006, pp.16-22.

[23] Alireza Hasaninejad, Abdolkarim Zare, Mohammad Reza Mohammadizadeh and Mohsen Shekouhy, ARKIVOC 2008 (xiii) 28-35.

[24] Khodabakhsh Niknam , Dariush Saberi and Maleki Mohagheghnejad, Molecules 2009, 14, 1915-1926.

[25] Debasish Bandyopadhyay, Sanghamitra Mukherjee, Robert R. Rodriguez and Bimal K. Banik, Molecules 2010, 15, 4207-4212.

[26] Hossein Reza Darabi; Kioumars Aghapoor; Farshid Mohsenzadeh; Mohammad Reza Jalali; Shiva Talebian; Leila Ebadi-Nia; Ehsan Khatamifar and Ali Aghaee; Bull. Korean Chem. Soc. 2011, Vol. 32, No. 1, pp. 213-218.

[27] Rajesh S. Bhosale; Swapnil R. Sarda; Suresh S. Ardhapure; Wamanrao N. Jadhav; Sudhakar R. Bhusare and Rajendra P. Pawar; Tetrahedron Letters 46 (2005) 7183-7186.

[28] Wen-Xue Guo; Hui-Le Jin; Jiu-Xi Chen; Fan Chen; Jin-Chang Dinga and Hua-Yue Wu; J. Braz. Chem. Soc., Vol. 00, No. 00, 1-6, 2009. 
[29] Hossein Reza Darabi; Fatemeh Tahoori; Kioumars Aghapoor; Fatemeh Taala and Farshid Mohsenzadeh; J. Braz. Chem. Soc., Vol. 19, No. 8, 1646-1652, 2008.

[30] A. Hasaninejada; A. Zareb; M.R. Mohammadizadeha and Z. Karami; J. Iran. Chem. Soc., Vol. 6, No. 1, March 2009, pp. 153-158.

[31] Radhakrishnan Mahesh; Arghya Kusum Dhar; Tara Sasank T.V.N.V. ; Sappanimuthu Thirunavukkarasu; Thangaraj Devadoss; Chinese Chemical Letters 22 (2011) 389-392.

[32] Fang Dong; Gong Kai; Fei Zhenghao; Zhou Xinli; Liu Zuliang; Catalysis Communications 9 (2008) 317-320.

[33] ALIREZA HASANINEJAD; ABDOLKARIM ZARE; MOHSEN SHEKOUHY and AHMAD REZA MOOSA-
VI-ZARE; E-Journal of Chemistry, 2009, 6(S1), S247- S253.

[34] Mohammad Reza Islami and Zahra Hassani, ARKIVOC 2008 (xv) 280-287.

[35] Mohammad Ali Zolfigol; Hooshang Vahedi; Abdoulhossein Massoudi; Sami Sajjadifar; Omid louie and Ahmad Rez Moosavi Zare; Current Medicinal Chemistry 2011, pp. 202(3rd International Conference on Drug Discovery \& Therapy February 7- 10, 2011, Dubai Women's College, Dubai, UAE).

[36] Alireza Hasaninejad, Abdolkarim Zare, Mohammad Reza Mohammadizadeh and Mohsen Shekouhy, ARKIVOC 2008 (xiii) $28-35$

[37] Kaye, I. A.; J. Med. Chem. 1964, 7, 2. 